\title{
Blockchain and DLTs in the Maritime Industry: Potential and Barriers
}

\author{
A. Panos, G. Kapnissis, and H. C. Leligou
}

\begin{abstract}
In the shipping industry, a significant part of the documents exchanges still have the traditional paper form, mainly due to security concerns, despite the size and modernization efforts of this market. We explore the adoption of the blockchain and Distributed Ledger Technologies to address document exchange in a fast and secure way.
\end{abstract}

Index Terms - Bill of Lading, Blockchain, DLT, Maritime Industry.

\section{INTRODUCTION}

The digitalization of the shipping industry lags behind many other sectors of the economy even though it is responsible for the carriage of around $90 \%$ of world trade and is the backbone of world commerce [1]. Although many aspects of the shipping industry have been modernized and attempt to create unmanned vessels that have attracted the research attention, the digitization (dematerialization) of the document exchange activity that accompanies the vessel and the load has not witnessed significant progress. Several attempts have been made since digitizing this process is expected to save large amounts of money and human and paper resources worldwide. An example, there are cases where the cargo arrives at the port and the relevant (paper) documents are not yet available at the destination port introducing high costs. The dematerialization attempts and their wide adoption has been hindered up to now primarily due to the reluctance of the involved actors to embrace them raising security concerns. They consider that keeping the documents in a server where multiple actors have access makes this server prone to attacks by adversaries. Additionally, mutual trust among the diverse actors cannot be assumed which triggers concerns about data integrity and modification.

The advent of blockchain technologies radically changes the landscape of secure data and document exchange affecting almost all sectors of the economy. While this technology has initially been proposed for financial exchanges (attempting to substitute the current banking system or run in parallel), it exhibits very important characteristics exploitable in many different cases. This technology mandates the storage of data in multiple devices across the world so that even if one of these "devices" is attacked, the data remain available. Another interesting characteristic is that once any piece of information is stored in the blockchain, no device/user can go back and alter it. Third (and not at all last), the participating entities establish trust among themselves since to participate in this solution they have

Published on October 7, 2020.

A. Panos, University of West Attica, Greece.

(corresponding e-mail: apanos@uniwa.gr)

G. Kapnissis, University of West Attica, Greece.

(e-mail: kapnissis@hotmail.com) to somehow "pay" or be approved to participate so any entity attempting malfunctioning is traceable and can be identified and accused.

In this article, we present processes of the maritime industry that would benefit from their dematerialization. Then we proceed to review solutions that have been proposed to address the dematerialization processes and then turn our attention to blockchain-enabled solutions and the benefits they bring. We describe how Blockchain and Distributed Ledger Technologies can bridge the gap and help realize the transition from the paper to the digital form of Bill of Lading (BOL), which is one of the most important processes in the shipping industry. We also explore the use of different types of DLTs and present the few commercial solutions. Finally, we assess the current solutions towards identifying the current gaps and discuss the obstacles that the adoption of such a solution would face.

Our goal and contribution is twofold: a) to explain to maritime industry people and leaders why blockchain enabled solution is secure and reliable and can bring them all the benefits of a digitized process and $b$ ) to guide the ICT industry create a blockchain enabled solution that perfectly matches the needs of the maritime industry.

\section{MARITIME INDUSTRY PROCESSES}

The operation of the maritime industry entails the execution of multiple processes that ensure appropriate, timely, and sale delivery of all kinds of goods around the world, through a complex and international ecosystem. In the sequel, we examine three processes that are currently performed through paper, are very bureaucratic, time-consuming, and thus inflate the transportation costs.

\section{BILL OF LADING}

One of the most significant documents in international commercial transactions, which has been an integral component in the maritime shipping industry, is the bill of lading (BOL). It was the result of the inability to ensure that goods were delivered by the carrier to the correct consignee and also arrived at the consignee in the same quantity and quality as when delivered by the producer, consignor, or exporter, to the carrier.

Regardless of the types of BOL, as a document, to be considered valid, it must serve three basic functions, necessary for the separation of legal obligations and commitments between the parties:

H. C. Leligou, Univeristy of West Attica, Greece.

(e-mail: e.leligkou@ uniwa.gr). 
1) It must be a conclusive receipt, i.e. an acknowledgment that the goods have been loaded

2) It must contain or evidence the terms of the contract of carriage, and

3) It must serve as a document of title to the goods, subject to the "Nemo dat quod non habet" rule (literally meaning "no one gives what they don't have").

In addition to the above, various types of BOL have some additional features that allow them to be transferred as a transferable document of title, to another person while transferring the right to acquire and receive the goods they represent.

In this paper, we will focus and analyze the Negotiable BOL, because it is the most known obstacle of BOL transformation (form paper to paperless) and, unfortunately, the most complex subcategory.

A negotiable bill of lading (N/BOL) is distinguished by the fact that it is a contract of carriage that can be transferred to a third party. Under that definition, and in conjunction with its functions, a negotiable bill of lading (N/BOL):

1) Operates as a receipt providing evidence that goods conforming to the contract have been shipped as agreed and are in the physical possession of the carrier for delivery to the consignee at the destination.

2) Contains or evidences the relevant terms of a contract with the carrier

3) Operates as a transferable document of title, and it is exactly this aspect, which makes it different from the non-negotiable bill of ladings.

While necessary for ensuring trust in international transactions, BOL significantly burdens the exchanges: Each BOL is often sent at least three times through a courier process, costing $\$ 100$ on average. With more than 50 million BOLs being created every year, the estimated total yearly cost of this process is approximately $\$ 5$ billion. Keeping BOL in paper format requires manual processing, increases the probability of being lost or forged. In maritime logistics, the shipper must mail physical copies of the bill to the importer of the goods. If the goods reach the importer in advance of BOL, the importer will not have the required document of title to present to the carrier. Not only will the carrier not accrue liability — neither in trover nor under the statute - for withholding the goods, but the carrier may also have to place the goods in storage. This can result in demurrage costs as well as potentially causing other economic loss due to fluctuations in the market value of the goods or an inability of the importer to meet obligations under other contracts [2]. It is evident that in this process multiple actors are involved including carriers, consignees, and others.

The above reasons, along with the advent of the internet, the development and spreading of electronic information systems, have largely led to the development of electronic solutions for the processing and transaction of BOLs also known as EDI (Electronic Data Interchange). The most significant examples approved by Clubs Are Bolero, essDOCS, e-title, and edoxOnline.

Several problems have emerged from this effort and as a result, we had a limited acceptance and adoption with an inability to replace the pre-existing situation with the new one. The most important of these [3] are presented below and are based both on systems analysis and research among users (carriers, consignors, and consignees):
1) Commodity traders did not like to have their transactions recorded in a central registry.

2) The ultimate buyers of commodities did not want to acquire bills of lading from a registry supporting and servicing intermediaries and speculators.

3) The liability of e-Bill of Lading platforms was not established. This resulted in relatively expensive insurance contracts for the registry operations.

4) If the goods are to be sold to a party not being a member of the specific e-Bill of Lading platform, the e-Bill of Lading may not be used anymore. In such a case the parties may have to switch to a paper bill of lading to be issued at that moment by the carrier on the request of the actual consignee.

5) All Contracting Parties must be members of this e-Bill of Lading platform and have signed and accepted the terms and conditions of this platform.

6) The platforms leave it on the responsibility of its users to assess if they comply with the laws of the country the user wishes to trade in or with. As the solutions are based on contractual relationships, legal uncertainty remains as to the acceptance and enforceability of the system in various jurisdictions.

7) In most cases, the platforms required, the trade transaction to be financed by a letter of credit.

8) Not all banks (which are a key factor in the equation of transactions) accept an e-BOL as a warrant to credit or withdraw money from an account.

9) Lack of international legal framework.

10) There must always be a trusted third party.

Beyond the literature review, which concludes to the above factors, the United Nations Conference on Trade and Development researched the involved parties, to identify from the practical point of view, the obstacles using electronic platforms, and conclude that the top four obstacles are: Infrastructure/market/trading partners not yet ready; the legal framework is not clear enough or is not adequate; electronic equivalents are not sufficiently secure; technology and/or switch to the electronic environment is too costly.

Analyzing the spotted factors, we note that we have two different types of obstacles. The first type includes legal issues and the second, technology issues which generate a chain of more obstacles.

\section{CHARTERING}

The Charter is the basic contract of carriage. The terms of the Charter are determined by the obligations and rights of the parties and the chartering agreement is considered valid in any way and formulated to deal with all the issues that may arise as much as possible. For example, English law agreement has Expressed terms, implied terms, representations, conditions, warranties, and innominate terms. Different forms of brokering agreements are distinguished like standard Charter, agreed charter parties, adopted charter parties, Recommended charter parties. The approved charter parties, depending on Chartering Type are Voyage Charter, Time Charter, and Bare Boat Charter. 


\section{PORTS ClEARANCE}

Ports play a significant role in Maritime Industry, as freight must be loaded or departed on port stations and automation plays a key aspect to provide quick and efficient service. All vessels are obliged to obtain a Port Clearance Certificate (PCC) before departing or arriving at a specific port. The departure declaration, together with the relevant documents, must be made to the Maritime and Port Authority (MPA) to obtain the PCC. It is obvious that to obtain the port clearance certificates for a specific journey, multiple entities (port authorities and vessel owners/companies) are involved. Performing this process in a paper-based manner is inefficient (time and resource consuming).

\section{BLOCKCHAIN AND DLT TECHNOLOGIES}

Blockchain technology is indisputably at its hype. Blockchain is a technology that implies that a) several nodes form a "blockchain network", b) all these nodes keep a replica of a database, c) the database is constructed in the form of chains of blocks where each block consists of multiple "transactions" and each block "points" of the older ones in the chain with the pointer being calculated as a hash function of the data included in the block. To motivate the nodes, perform the calculations, specific rewards are granted to the nodes/miners and the nodes implement a specific consensus mechanism to agree on the next block to be inserted in the chain.

The major benefits stemming from these basic principles are:

- The system is immune to single node attacks. If one of them is attacked, the network remains active, because the information is kept in multiple nodes;

- Immutable information- information integrity: nobody can alter a piece of information already stored in the blockchain, as this is protected by the hash which acts as a pointer to the block; changing the information in a block would require the alteration of all blocks from the altered one onwards from all nodes, which is practically impossible;

- Distributed operation of the information maintenance solution which implies an equivalent distribution of the operational and capital expenditure costs.

The popularity of blockchain technology has led to articles proposing its adoption in almost all sectors including private, public, energy, financial, record keeping, identity management among others. However, blockchain is not a panacea and has to be carefully applied to provide solutions where its benefits justify its cost which is mainly due to the computational intensity.

When trying to design a blockchain-based solution, different design alternatives exist and the solutions architects have to make the relevant decisions:

- Private vs. public and permissioned vs. permissionless: There is the option to create a public blockchain where anybody can join or a private blockchain where only nodes authorized by a certain authority can enter or a federated /consortium blockchain where a consortium agrees in the establishment of a network. The chosen type of blockchain is also affecting the selection of the consensus mechanism as well as the transaction speed. Known Networks for those types of blockchain are
Ethereum (Public), Hyperledger Fabric (Private), Libra (Consortium).

- The structure of the information to be kept in the blockchain is also a design consideration. The "transaction" originally kept in the blockchain can be restructured to accommodate other information or even to store pointers to files storage systems like IPFS (interplanetary file system) which is more likely to be the case in the maritime industry as well.

- Structure of the "database": while in blockchain, the information is stored in chains of blocks, there are more than ten platforms that implement other information structures. Other platform organizes the information in "blocks" of just one transaction or organizes the blocks in Directed Acyclic Graphs (and not chains). All these approaches (including blockchain) fall under the umbrella of Distributed Ledger Technologies. Despite the fact, the blockchain is a sub-category of DLTs, the technology as a whole is widely known as the blockchain.

- Definition of the architecture of the solution: i.e. definition of where the mining nodes are deployed, who owns them, where the applications to access the network are running, and others. Apart from the positioning and ownership of the nodes, their different parameters have to be defined (such as consensus algorithms, platformspecific parameters like channel organization in Hyperledger Fabric, e.t.c.)

It is worth clarifying that in the sequel we refer to "platforms" as approach and open source code that could be used to develop a specific application/solution while "solution" is the software that a company with the install and deploy in and for a shipping company, i.e. it is tailored to the specific use

\section{DLT SOLUTIONS AND BILL OF LADING}

Currently, the solutions that embrace blockchain-distributed ledger technologies addressing the bill of lading operations are limited and mainly come from the broader category of solutions for the supply chain.

The CargoX dApp [4] is a web-based decentralized application allowing customers to interact with Smart BOL digital documents. CargoX is an Independent Supplier of Blockchain-based Solutions for Logistics. The solution that the enterprise provides is based on the public blockchain Ethereum Network and uses the CargoX B/L exchange Protocol with the ERC20 CXO token that serves as a protocol utility token and drives the core functionalities of CargoX's smart contracts.

At the moment, CargoX can only provide to the customers a House Bill of Lading (HBL) which is a transportation contract between a Non-Vessel Owning Common Carrier (NVOCC) /Freight Forwarder and an end customer (the shipper). As such, it cannot be used as a Negotiable Bill of Lading. Even if CargoX uses blockchain and in some ways can send e / BOL to different parties, it is approved by the International Group of P\&I Clubs.

The second solution is essDocs Voltron which is the leading enabler of paperless trade, announced that it has agreed to integrate its CargoDocs DocEx solution into the blockchainbased Voltron application. Voltron - developed on R3's Corda platform - provides a multi-bank channel for companies to 
digitally manage the issuance of Letters of Credit (LCs) and electronic presentation of trade documents, including title documents such as Bills of Lading (BOLs). Corda Enterprise platform is an open-source platform designed for individual firms to interoperate with those who are using open source. EssDocs gives access to banks and corporates to all data (plus documents) within the Voltron Platform. This collaboration between essDocs and Voltron aims to create a community with total access to all data and tradeable offers for everyone. It is more like a cross-platform solution. EssDocs are similar to Bolero as a standalone centralized transferable e B/L platform. By collaborating with Voltron, it wants to give access to banks using its data for a more efficient bill of lading contract.

Corporate giants such as IBM and MAERSK, apply blockchain technology with the introduction of TRADELENS which corresponds to a broader supply chain process and not purely shipping activity (Charter, Bill of lading). It is based on IBM's project called Hyperledger Fabric (HLF) OASIS, which is a private Blockchain that does not use any form of cryptocurrency. The goal of this solution is to empower businesses and authorities along the Supply Chain with a single, secure source of shipping data, enabling more efficient global trade. The solution consists of three layers: a) the network layer: that consists of network shippers, forwarders, ports, and terminals that together bring a wealth of information that can be tracked, stored, and shared across the ecosystem; b) the Platform layer: that is located above of the network's data and manifests itself into a dynamic, functional medium. This open platform is underpinned by HLF Blockchain, creating an end-to-end audit trail of important shipment information and a secure vehicle to share critical documentation; c) Application and services layer: which leverages the network and capabilities of the platform. Tradelens follows the consensus of Hyperledger Fabric.

EdoxOnline approved by the International Group of P\&I Clubs, allows an automatic issue and manages to eBOLs in collaboration with supply chain members. It is an eBOL platform using Blockchain Technology where document elements are created and entered from the destination point and sent to exporters in the origin country. Exporter coordination with other members of the supply chain is needed. It is using two-factor authentication and the system provides the required information to each member.

Bolero is the oldest existing eBOL system. The full name is Bill of Lading Electronic Registry Organization (Bolero). It provides other supply chain documentation like purchase orders, invoices, letters of credit, insurance certificates, and more. Its main feature is named Rulebook (the legal framework guarantees the document exchange on the platform are valid and encrypted). An electronic bill of lading can be created only by a carrier, adding cargo description and other bill of lading elements. By using this application, we can create an electronic bill of lading or scanning an existing one. It also provides TRI (title Registry Instruction) which is the record that ensures that data can't be altered or copied.

E-title is a peer to peer platform, characterized as a hybrid platform accepting all document formats. It is established as a system where you can have a smooth workflow regardless of the type of document formats your partner use (pdf, image, XML, EDI). A carrier creates a bill of lading on paper and transfers it to e-title. Then eBOL created, signed, and then registered to e- titles Hardware Security Modules. It operates following UNCITRAL model law.

\section{ASSESSMENT AND DISCUSSION}

\section{A. Technical Aspects}

A first assessment of the presented solutions is included in table 1 where the type of each solution and its main advantages and disadvantages are listed. Any solution needs to deploy a private or permissioned platform so that access to the information is fully controlled. From the access control perspective, Hyperledger Fabric-based solution is advantageous because it is a private network build with inter-enterprise exchanges in mind. For this purpose, it supports the notion of "channels" where nodes communicated in the same "channel" essentially form a blockchain network. This way deploying HLF based solutions, multiple blockchain networks can run in parallel. This capability gives a purely operational form to the network that makes it more appealing to shipping organizations. For example, the different channels could be exploited to segregate groups of shipping organizations. On the other hand, Corda is more tailored to financial processes that are indispensable to any shipping operation.

As the need to register and maintain continuously growing amounts of information is evident, scalability plays a crucial role. Scalability refers to the capacity of the solution to register and manage larger amounts of information (at an adequate speed to avoid the degradation of the user experience) and unfortunately, scalability is an inherent drawback of blockchain approaches. Additionally, today the trend is to register continuously increasing amounts of information for all goods in an attempt to offer enhanced security and added value services to the end-users. Across all supply chains, actors seek to provide evidence to end-users that the goods are appropriately handled and safe to use. This is nowadays feasible through the integration of sensor systems that are capable of collecting information across the journey of the goods installed in all places where the goods are placed. The need for handling continuously increasing amounts of information due to a) the ordinary evolution and $b$ ) the integration of additional information sources render scalability of paramount importance. Turning our attention to the scalability features of the existing solutions, scalability is not among the advantages of Ethereum. Hyperledger Fabric exhibits better scalability than Ethereum as it can organize the blockchain network in channels which could be seen as independent networks. Currently, it is DLTs that come at a rescue and exhibit better scalability features. For example, directed acyclic graph approaches come with higher scalability potential which of course depends on the way they are structured, and they operate. 
TABLE I: BLOCKCHAIN-BASED E-BOL PLATFORMS AND SOLUTIONS

\begin{tabular}{|c|c|c|c|}
\hline & Type & Advantages & Disadvantages \\
\hline CARGOX & $\begin{array}{l}\text { Public (restricted) } \\
\text { Ethereum-based } \\
\text { Blockchain }\end{array}$ & \begin{tabular}{|} 
Well Known \\
blockchain House of \\
Lading \\
Transactions are \\
viewable to the entire \\
network \\
Helped blockchain to \\
be acceptable in \\
Shipping Industry
\end{tabular} & $\begin{array}{l}\text { Does not support } \\
\text { negotiable BOL } \\
\text { Not approved by } \\
\text { P\&I clubs } \\
\text { High Value of } \\
\text { tokens make } \\
\text { transaction } \\
\text { unacceptable }\end{array}$ \\
\hline $\begin{array}{l}\text { EssDocs- } \\
\text { Voltron }\end{array}$ & $\begin{array}{c}\text { Permissioned Based } \\
\text { on Corda }\end{array}$ & $\begin{array}{c}\text { Cross-platform } \\
\text { connectivity } \\
\text { Use R3 Corda } \\
\text { Ricardian Contract }\end{array}$ & $\begin{array}{c}\text { Beta mode } \\
\text { Finance based } \\
\text { model } \\
\text { Banks have access } \\
\text { to all companies' } \\
\text { data } \\
\text { Cannot use a token }\end{array}$ \\
\hline Tradelens & $\begin{array}{c}\text { Private (based on } \\
\text { Hyperledger Fabric) }\end{array}$ & $\begin{array}{c}\text { The generic platform } \\
\text { for supply chain } \\
\text { solutions } \\
\text { Promoted by IBM } \\
\text { and Maersk }\end{array}$ & $\begin{array}{l}\text { Doesn't provide } \\
\text { maritime specific } \\
\text { operations. }\end{array}$ \\
\hline e-title & $\begin{array}{c}\text { Peer } 2 \text { peer } \\
\text { Hybrid platform }\end{array}$ & $\begin{array}{l}\text { Accept any format of } \\
\text { Document (PDF, } \\
\text { IMAGE, XML, EDI) }\end{array}$ & $\begin{array}{c}\text { The need of Paper- } \\
\text { Based bill of lading } \\
\text { to convert it in } \\
\text { electronic Bill of } \\
\text { Lading } \\
\end{array}$ \\
\hline Edox Online & $\begin{array}{l}\text { Web-based } \\
\text { blockchain }\end{array}$ & P\&I Clubs accepted & Lack of privacy \\
\hline Bolero & Cloud-Based & $\begin{array}{c}\text { Scan paper } \\
\text { documents } \\
\text { Manage a Huge load } \\
\text { of Documents }\end{array}$ & $\begin{array}{c}\text { Contractual } \\
\text { provisions binding } \\
\text { only the } \\
\text { contracting parties } \\
\text { and cannot provide } \\
\text { obligations to third } \\
\text { parties } \\
\text { Centralized }\end{array}$ \\
\hline
\end{tabular}

Let's now focus on the capacity of the solution to expand and interconnect with other enterprise systems like the RFID or sensor infrastructures installed in the vessels. This depends on the modularity or flexibility of the adopted platform/solutions to integrate. Hyperledger Fabric has been built with modularity in mind. Corda (on which essDocs is built) is consciously designed as DLT for the Banking and Financial Industry, hence, the main focus is on financial services transactions. Its architectural design is simple when compared to Fabric. As the framework of Fabric is modular it can likely be altered to resemble Corda. There are efforts as of now only which seek to integrate Corda into the Hyperledger project. In that sense, currently, Corda and Hyperledger Fabric cannot be seen as competitors but rather as a complementary platform. Additionally, CargoX and Bolero offer APIs (Application Programming Interfaces) facilitating integration with other platforms while e-title does not seem to offer such an option.

As these solutions come at a significant cost, solutions tailored to the needs of smaller businesses can be developed and offered. For example, a shipping company owning a few ships finds it expensive to acquire CargoX solutions for its needs. This may open a new niche where smaller IT companies come into the play and can use available platforms to build solutions tailored to the needs of smaller businesses. In this perspective, CargoX is more a platform (which another company could use to develop a specifically tailored solution) than a solution. It is important to understand that in this huge market, there cannot be a one-size-fits-all solution; it is more likely that many solutions embracing the same principles of operation are in place and can work together.

TABLE II: PRINCIPLES OF UNICTRAL MET BY PRE-DLT AND DLT-BASED SOLUTIONS

\begin{tabular}{|c|c|c|}
\hline $\begin{array}{c}\text { UNICITRAL Model Law on } \\
\text { Electronic Transferable Records } \\
\text { Requirement [5] }\end{array}$ & Pre-DLT Efforts & $\begin{array}{c}\text { DLT-Based } \\
\text { Solutions }\end{array}$ \\
\hline Electronic Signature & YES & YES \\
\hline Uniqueness and singularity & NO & YES \\
\hline $\begin{array}{c}\text { Contains the information that would } \\
\text { be required to be contained in a } \\
\text { transferable document or instrument }\end{array}$ & YES & YES \\
\hline $\begin{array}{c}\text { Control: Establish exclusive control } \\
\text { of that electronic transferable record } \\
\text { by a Person. Identify that person as } \\
\text { the person in control }\end{array}$ & NO & YES \\
\hline $\begin{array}{c}\text { Retain the integrity of the electronic } \\
\text { record }\end{array}$ & NO & YES \\
\hline $\begin{array}{c}\text { Ability to prevent unauthorized } \\
\text { access to and use of the System }\end{array}$ & YES & YES \\
\hline
\end{tabular}

Under the pressure of technological innovation, the need to promote merchant trade, economic growth, and the legal support that now exists, Bahrain has been enacted the MLETR [6]. Czech and Singapore have also conducted public consultations on its adoption. Another public consultation has been conducted in Singapore in the summer of 2019, in the broader framework of the review of the Electronic Transactions Act. Further details on legislation for blockchain in shipping can be found in [7]. Any proposed solution for electronic transferable records should initially meet the requirements of the MLETR. Additional rules of law may be adopted and supplemented to be universally recognized and accepted and the electronic version of a traditional document 'shall not be denied legal effect' (MLETR Art 7(1)). According to the MLETR, electronic transferable records that are capable of replacing the current paper-based documents, and in particular an electronic form of bill of lading, should be based on a technology that can meet the requirements of the legal framework and Hyperledger Fabric can provide a legal framework through Smart Contracts and Ricardian Contracts.

The legislative framework alone is not capable of overcoming the obstacles that exist about the implementation of the electronic circulation of title documents. Another important step to be taken is the creation of a specialized court, properly staffed by judges specialized in maritime law and with appropriate technological know-how, so that they can resolve claims and disputes arising from the electronic version of a traditional document of title.

\section{CONCLUSION}

The maritime industry is a sector where dematerialization lags behind other sectors and is still using traditional methods to a high extent to do business. The blockchain and distributed ledger technologies enable the design and development of solutions that fit all requirements of this sector as its main characteristics refer to security and privacy control. We reviewed existing solutions trying to identify also rooms for improvement and draw guidelines for prospective designers of new solutions. Major conclusions are that the DLT-based solutions have to ensure scalability because the amount of 
information and information sources is continuously and rapidly increasing and that meeting the mandates of the current legislation is crucial and should not at all be underestimated. Policymakers, on the other hand, should be aware of the situation and flexible to adapt and integrate novel technologies in the quiver of the sector. Comparing the proposed commercial DLT-based solutions of BOLs, we consider that current solutions need to be improved. Future researchers will be able to design an integrated platform that will cover and solve all the limitations and obstacles, identified above, to facilitate the maritime partners to digitize BOLs, as well as the use of Distributed Ledger Technologies on Chartering.

\section{ACKNOWLEDGMENT}

This work was supported in part by the University of West Attica's internal research project.

\section{REFERENCES}

[1] Sanchez Gonzalez, Pedro Luis \& Díaz-Gutiérrez, David \& Leo, T.J. \& Núñez, Luis. "Toward Digitalization of Maritime Transport?", Sensors. Vol. 19, no. 4, pp. 926. 10.3390/s19040926, (2019).

[2] Jake Herd, 'BLOCKS OF LADING' Distributed Ledger Technology and the Disruption of Sea Carriage Regulation, QUT Law Review - Vol 18, No 2 .

[3] Raphael Brunner, Electronic Transport Documents And Shipping Practice Not Yet A Married Couple, LL.M. Shipping Law, 2007.

[4] https://cargox.io/CargoX-Business-Overview-Technology-Bluepaper.pdf.

[5] UNCITRAL Model Law on Electronic Transferable Records.

[6] Takahashi, Koji. "Bahraini legislation based on the UNCITRAL MLETR". Blockchain, Cryptocurrency, Crypto-asset and the Law. Retrieved 12 February 2019.

[7] Liu H. (2020) Blockchain and Bills of Lading: Legal Issues in Perspective. In: Mukherjee P., Mejia, Jr. M., Xu J. (eds) Maritime Law in Motion. WMU Studies in Maritime Affairs, vol 8. Springer, Cham.

[8] United Nations Conference on trade and development, the use of transport documents in international trade, UNCTAD (2003).

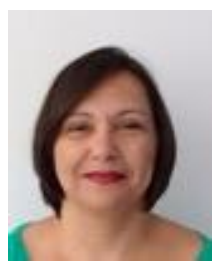

Dr. Helen C. (Nelly) Leligou received her Dipl.-Ing. and $\mathrm{PhD}$ from the Electrical Engineering and Computer Science Department of NTUA, Greece in 1995 and 2002 respectively. She is currently (from Oct. 2017) associate professor at University of West Attica while from 20072017, she acted as assistant professor at Technological Educational Institute of Sterea Ellada.

Her research interests lie mainly in the areas of Information and Communication Technologies including a) routing protocols and trust management in Wireless Sensor Networks, b) control plane technologies in broadband networks including HFC, PON, WDM metro and core networks and c) industrial, embedded and network system design and development. Currently she is working on block-chain technologies, IoT enabled systems and gamification for various application sectors including social innovation, education and participatory approaches. She is the scientific coordinator of the H2020-ASSET project. Her research results have been published in more than 100 scientific journals and conferences. She has participated in several EUfunded ACTS, IST, ICT and H2020 research projects in the above areas. She also acts as evaluator for $\mathrm{H} 2020$ proposals. She is also a visiting professor at the Hellenic Open University.

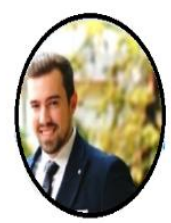

PhD Candidate Panos Apostolos received his MSc on Maritime Industry, Transportation and Public Affairs from University of Aegean (2019). Before that, he received a BSc on Economics by National and Kapodistrian University of Athens (2017).

His research interests lie mainly in the areas of New Technologies on Maritime Industry and Education including a) Robotics, b) DLTs c) IoT Devices. He works on Open Robotics Platforms to create interesting projects for students. He works as an ITBM Administrator on Finance Sector and served as a Sergeant on Marine Corps.

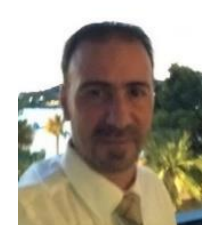

George P. Kapnissis was born in Athens (Greece) in 1976 $\mathrm{He}$ is an Officer (Lieutenant Commander) of the Hellenic Navy. Holds a Bachelor's degree in Naval Science from the Hellenic Naval Academy (1999) and an MSc in New Technologies in the Maritime Industry and Supply Chain from the University of West Attica and Aegean University (2020).

Currently, and after a long period of deployment on combat ships, he is the Director of Combat Systems and Ammunitions of the Hellenic Fleet Command. His research interests lie mainly in the areas of New Technologies in the Maritime Industry and Technology acceptance models and he does research in Blockchain and DLTs development and adoption, applying Network Theory and Qualitative Social Research. 